\title{
Reduced KISS1 expression acts as a predictor for aggressive features of cervical carcinoma and promotes migration and invasion in cervical carcinoma cells
}

\section{Zhuo Wang}

Jilin Cancer Hospital

Min Wang

Jilin Cancer Hospital

Hao Yu

Jilin Cancer Hospital

Hongyu Wang

Jilin Cancer Hospital

Can Wang

Jilin Cancer Hospital

Jian Ou

Jilin Cancer Hospital

Fanxu Meng

Jilin Cancer Hospital

Yang Zhang ( $\nabla$ yzjldx@126.com )

Jilin Cancer Hospital

\section{Research}

Keywords: KISS1, cervical carcinoma, metastasis, proliferation, migration, invasion

Posted Date: April 28th, 2020

DOI: https://doi.org/10.21203/rs.3.rs-24040/v1

License: (c) (i) This work is licensed under a Creative Commons Attribution 4.0 International License. Read Full License 


\section{Abstract \\ Background}

Cervical carcinoma causes high morbidity and mortality in patients largely due to its invasion and metastasis. Kisspeptin (KISS1) had been found as metastasis suppressor in many malignancies. But the clinical significance and biological functions of KISS1 in cervical carcinoma was not elaborated.

\section{Methods}

The expression levels of KISS1 were detected by quantitative real time polymerase chain reaction (qRTPCR), Methylation specific PCR (MSP) analysis and western-blotting in cervical carcinoma tissues and cells. A eukaryotic expression plasmid was transfected into cervical epithelial cells in order to initiate KISS1 overexpression, and the endogenous was silenced in cervical epithelial cells using short hairpin RNA (shRNA) sequences. Transfection efficiency was validated by western blotting assays. To explore the effects of KISS1 on the metastatic phenotype of cervical carcinoma, the cell proliferation was examined by CCK8 assay, and the cell migration and invasion were detected by cell scratch and transwell invasion assays, respectively.

\section{Results}

We investigated the relevance between KISS1 expression with its methylation and clinicopathological features of cervical carcinoma. Low expression of KISS1 predicts a poor prognosis and was associated with lymph node metastasis and TNM stage. And methylation of KISS1 promoter, which was linked with KISS1 inactivation, was also found in most clinical samples of aggressive cervical carcinoma. In vitro cervical carcinoma C33A cells, KISS1 overexpression significantly inhibited cell proliferation, migration and invasion in cells. While KISS1 knockdown promoted proliferation, migration and invasion in cervical epithelial cell line CRL-2614.

\section{Conclusion}

In summary, low-expression of KISS1 play a suppressive role for proliferation, migration and invasion in cervical carcinoma cells. Its expression and methylation are associated with clinical progression of cervical carcinoma. Hence, KISS1 could be a potential diagnostic and prognostic marker as well as a new target for the treatment of cervical carcinoma.

\section{Introduction}

Cervical cancer is characterized a malignant tumor originating from the cervical epithelium, which mainly metastasizes to the surrounding tissues, organs and lymph nodes via local invasion and infiltration [1]. 
Although various comprehensive treatments including surgical resection, radiotherapy, and chemotherapy have been used at different stages of the disease, the incidence, and mortality of impaired quality of life are still rising $[2,3]$. Therefore, it is necessary to explore new biomarkers and therapeutic targets that will help develop targeted therapies for cervical carcinoma.

Metastasis suppressor genes play a key role in tumor metastasis [1]. A previous study demonstrated that the effect of metastasis suppressor genes on tumor metastasis was more critical than that of metastasis promoter genes, and the reduction in the expression of metastasis suppressor genes or gene loss might induce the invasion and metastasis of tumor cells[4]. KISS1 was initially identified as an important tumor metastasis suppressor gene in human melanoma cells[5]. KISS1 is located on the long arm of human chromosome-1. The protein-encoding gene acts as an endogenous ligand for G-protein coupled receptor 54 (GPR54) and produces a variety of physiological effects, including inhibition of tumor cell proliferation, metastasis, invasion, and induction of tumor cell differentiation and apoptosis[6, 7]. The decrease in KISS1 levels and its role in tumor invasion and metastasis has been evaluated in various tumors, such as the bladder[8], colorectum[9] and breast cancer[10]. However, the expression of KISS1 and its pathogenesis in cervical carcinoma remains to be elucidated.

This study examined the methylation status and expression level of KISS1 in cervical carcinoma tissues, and then assessed the association between KISS1 methylation, KISS1 expression and clinical clinicopathological features. The effect of KISS1 on the biological function of cervical carcinoma cell lines was also studied. Our goal was to investigate the role of KISS1 in the development and progression of cervical carcinoma and whether it could prevent and treat clinical cervical carcinoma.

\section{Materials And Methods}

Patients and specimens. 97 cases of human cervical carcinoma samples, 12 cases of intraepithelial neoplasia (IN) tissues and 92 cases of nonneoplastic normal tissues were collected the time of surgical resection at the Jilin Cancer Hospital from July 2014 to July 2016. Samples were snap-frozen in liquid nitrogen and stored at $-97^{\circ} \mathrm{C}$ from 97 cervical carcinoma patients including with a median age of 65 (range, 51-75 years); Paraffin-embedded tissues were prepared by the Department of Pathology at the same hospital from the 97 cervical carcinoma patients with a median age of 62 (range, 34-82 years). Informed consent was obtained from each patient before collection of tissues. All diagnoses of cervical carcinoma were confirmed by histopathological examination. Information about patients and disease characteristics came from a review of the patient's medical records. The study was approved by the Institutional Ethical Review Committee of Jilin Cancer Hospital and adhered to the principles of the Declaration of Helsinki.

Cell lines culture. Human cervical carcinoma cell lines (C-33A, HeLa, SiHa, CaSki, HT3) and human epithelial HPV-16 E6/E7 transformed cervical epithelial cell line CRL-2614 were bought from American Type Culture Collection (ATCC; Manassas, VA), and cultured in F-12 k and RPMI 1697 (Gibco, Gaithersburg, MD, USA) supplemented with $10 \%$ fetal bovine serum (FBS), $100 \mu / \mathrm{ml}$ penicillin, and 
$50 \mathrm{mg} / \mathrm{ml}$ streptomycin, incubated at $37^{\circ} \mathrm{C}$ with a humidified atmosphere of $5 \% \mathrm{CO} 2$. Cells were identified by STR and no cross-contamination from other cell lines.

Methylation analyses of the promoter of KISS1. The primers: methylated KISS1 forward, 5'AAAGTTTCGTTTCGGAGGGTTC-3' and reverse, 5'-CTTTTATAAAACCCGAAATAACG-3'; and unmethylated KISS1 forward, 5'-AAAGTTTTTTTTGGGGGTTT-3' and reverse, 5'-CCTTTTATAAAACCCAAAATAACA-3'. Genomic DNA was extracted using standard methods. At the time of running electrophoresis, methylation positive control (CpG methylation enzyme modification after fresh placenta tissues DNA as a template), the unmethylation positive control (fresh placenta tissues DNA as a template) and the negative control $\left(\mathrm{H}_{2} \mathrm{O}\right)$ were set. The reaction conditions: pre-denaturation at $95^{\circ} \mathrm{C}$ for $10 \mathrm{~min}$, denaturation at $95^{\circ} \mathrm{C}$ for 45 $\mathrm{sec}$, annealing for $45 \mathrm{sec}$ (methylation-specific primer amplification annealing temperature $59^{\circ} \mathrm{C}$, nonmethylation specific primer amplification annealing temperature $\left.55^{\circ} \mathrm{C}\right), 72^{\circ} \mathrm{C}$ extension $50 \mathrm{sec}$, set the loop 34 times, and the final extension at $72^{\circ} \mathrm{C}$ for $7 \mathrm{~min}$. Agarose gel electrophoresis and staining were then performed. Data from the gel was collected using a under a UV transilluminator (ALPHA, San Leandro, USA) and subsequently analyzed.

Detection of the expression of KISS1 proteins by immunohistochemistry (IHC). Formalin-fixed, paraffinembedded sections $(4 \mu \mathrm{m})$ were placed into a $60-65^{\circ} \mathrm{C}$ box overnight and deparaffinized in green transparent agent, rehydrated with an alcohol gradient, and washed briefly in distilled water. To retrieve antigenicity, sections were boiled in $0.01 \mathrm{M}$ citrate buffer $\left(\mathrm{pH} \mathrm{6.0)}\right.$ ) for $20 \mathrm{~min}$ at $100^{\circ} \mathrm{C}$ and were washed in PBS 3 times after cooling to room temperature. Endogenous peroxidase was blocked with $3 \% \mathrm{H}_{2} \mathrm{O}_{2}$ (Bohai, Hebei, China) for 30 min then washed in PBS 3 times. Next, sections were incubated with normal sheep serum for $30 \mathrm{~min}$ at room temperature. Subsequently, sections were incubated at $4^{\circ} \mathrm{C}$ overnight with rat Anti-KISS1 monoclonal antibody (1:100 dilution; cat. no. ab55384; Abcam, USA, China). The following day, sections were rinsed with fresh PBS and incubated with biotinylated secondary antibody working solution and horseradish enzyme labeled streptavidin (cat. no. SP-9002; ZSGB BIO, Beijing, China) working solution respectively incubated at room temperature for $30 \mathrm{~min}$. Finally, sections were stained with diaminobenzidine (DAB) (cat. no. ZLI-9032; ZSGB BIO, Beijing, China) for visualization signal.

Vectors, transfection of target gene. KISS1 plasmid and control overexpressing were transfected into C33A cervical carcinoma cells using Lipofectamine 2000 (Invitrogen, Carlsbad, CA, USA); KISS1 sh, Scramble were transfected into cervical epithelial cell CRL-2614. Transfection effect were identified by reverse transcription quantitative polymerase chain reaction (RT-qPCR) and Western blot analysis.

RNA extraction and RT-qPCR. Total RNA was extracted using Trizol (Invitrogen, Carlsbad, CA, USA), and cDNA was synthesized using PrimeScript ${ }^{\text {TM }}$ RT reagent kit (Takara, Beijing, China) according to the manufacturer's instructions. Subsequently, expression levels of KISS1 were quantified by the real time qPCR using AceQ ${ }^{\circledR}$ qPCR SYBR ${ }^{8}$ Green Master Mix (Vazyme, NJ, USA) in ABI7500 quantitative PCR. Gene expression was defined based on Ct values and expression levels of genes were normalized to that of the housekeeping gene GAPDH as the control. The $2^{-\Delta \Delta} \mathrm{Ct}$ method was used to calculate relative 
expression changes. The thermocycling conditions were as follows: $95^{\circ} \mathrm{C}$ for $5 \mathrm{~min}$, followed by 97 cycles of $95^{\circ} \mathrm{C}$ for $10 \mathrm{sec}, 60^{\circ} \mathrm{C}$ for $30 \mathrm{sec}$ and $95^{\circ} \mathrm{C}$ for $15 \mathrm{sec}, 60^{\circ} \mathrm{C}$ for $60 \mathrm{sec}, 95^{\circ} \mathrm{C}$ for $15 \mathrm{sec}$. KISS1 primers: forward: 5'-CTCACTGGTTTCTTGGCAGC-3'; reverse: 5'-CTGGCTTCCTCTCGGTGC-3'; GAPDH were used as internal control reference: forward: 5'-GAGTCAACGGATTTGGTCGT-3'; reverse: 5'-

CATGGGTGGAATCATATTGGA-3'.

Western blot analysis. Protein in the supernatant was extracted after transfection as previously described [11]. Approximately $20 \mu \mathrm{g}$ of total protein was separated by $10 \%$ SDS-PAGE and transferred to a polyvinylidene difluoride (PVDF) membranes (Millipore, MA, USA). The membrane was blocked with $5 \%$ skim milk in PBS-T (10 mmol/l Tris, $145 \mathrm{mmol} / \mathrm{NaCl}, \mathrm{pH} 7.2-7.4)$ for $2 \mathrm{~h}$ at room temperature and then incubated with mouse monoclonal antibody KISS1 (1:1000 dilution; cat. no. ab55384; Abcam, Shanghai, China) or mouse monoclonal antibody $\beta$-actin (1:3500 dilution; cat. no. 60008-1-1 g; proteintech, Wuhan, China) overnight at $4^{\circ} \mathrm{C}$. Anti-mouse IgG (1:2500 dilution; cat. no. A23910; Abbkine, Wuhan, China) was used and developed with a chemiluminescent substrate. Images were taken with Oddessy CLX infrared fluorescence scanning imaging system (LICOR, Nebraska, USA) and the intensity of the bands was analyzed by Image $\mathrm{J}$ software (National Institutes of Health, Bethesda, MD, USA).

Cell proliferation assay. The transfected cells were independently seeded into 96-well plates and cultivated for 24, 48, 72 or $96 \mathrm{~h}$. Then, the Cell Counting Kit-8 (MedChem Express, NJ, USA) was added to each well and incubated at $37^{\circ} \mathrm{C}$ for $3 \mathrm{~h}$. The absorbance at $450 \mathrm{~nm}$ was measured using a microplate reader (Promega, Madison, USA).

Transwell Matrigel invasion assays. Cell invasion were measured using $8 \mu \mathrm{m}$ Transwell chamber (Corning, MA, USA) as previously described [12]. To measure invasion, a chamber containing Matrigel (Corning, MA, USA) was used and operated by the above method. After $24 \mathrm{~h}$, the chamber was stained by diff-quick staining (BASO, Taiwan, China) and counted in five random fields, the number of cells was expressed as an average.

Wound healing assay. When the transfected cells reached a growth density of $85 \%$, scratched the confluent monolayers with a pipette tip to create a gap to simulate a wound and washed with PBS. The transfected cells were then cultured in RPMI 1697. Images of the plates were obtained under a microscope (Olympus, Tokyo, Japan) at 0, 24, $48 \mathrm{~h}$.

Statistical analysis. All results were shown as mean \pm SD and analyzed using GraphPad Prism 7 (GraphPad Software, San Diego, CA,USA). For the in vitro experiments, the differences were analyzed using the unpaired two-tailed t-test. For the experiments involving clinical tissues, the differences were analyzed using Pearson's chi-square test. $\mathrm{P}<0.05$ was considered statistically significant.

\section{Results}

Methylation specific PCR (MSP) analysis of KISS1 gene promoter methylation. The MSP data obtained from 97 individuals showed that hypermethylation of the KISS1 gene promoter was observed in $71.1 \%$ 
(69/97) of a cervical carcinoma tissue, but only in $8.6 \%$ (8/92) of the nonneoplastic non-tumor tissues (Fig. 1A). Chi-square test showed that the hypermethylation of KISS1 in cervical carcinoma was significantly associated with lymph node metastasis and TNM stage $(P<0.05$; Table 1$)$. However, there was no statistically significant correlation between KISS1 promoter hypermethylation and other clinicopathological variables such as age, and histological grade $(P>0.05$; Table 1$)$. In addition, the mRNA expression levels of KISS1 were furtherly accessed in cervical carcinoma tissues and nonneoplastic cervical tissues. As expected, the mRNA expression levels of KISS1 was lower in cervical carcinoma tissues compared with non-neoplastic cervical tissues (Fig. 1B). The expression of KISS1 protein in cervical carcinoma tissues obtained from 97 individuals was assessed by western-blotting and immunohistochemical staining (Fig. 1C). As shown in Table 1, the expression of KISS1 was lower in cervical carcinoma tissues compared with that in non-neoplastic cervical tissues. In additionally, we found that KISS1 was expressed in the cytoplasm of cervical carcinoma cells (Fig. 1D and Table 2). KISS1 staining was detected in $24.7 \%$ (24/97) of cervical carcinoma tissues, significantly fewer in nonneoplastic non-tumor tissues 88.0\% (81/92). Then, we analyzed the correlation between KISS1 expression and clinicopathological features in cervical carcinoma patients. The low expression of KISS1 in cervical carcinoma was significantly associated with lymph node metastasis and TNM stage $(\mathrm{P}<$ $0.05)$. There was no statistical relationship with other variables such as age and histological grade ( $P>$ 0.05; Table II). In additionally, as shown in Fig. 2, cervical carcinoma patients with positive KISS1 expression exhibited longer overall survival compared with patients with negative KISS1 expression.

KISS1 is downregulated in cervical carcinoma cell lines. To investigate the regulatory role of KISS1 in cervical carcinoma, it was first examined whether KISS1 are dysregulated in cervical carcinoma cell lines. The results of RT-qPCR and western blot analysis demonstrated that KISS1 were expressed at lower levels in five cervical carcinoma cell lines (C33A, HeLa, Siha, Caski and HT3) compared with those in the human cervical epithelial cell line CRL2614 (Fig. 3A and B).

Overexpression of KISS1 on proliferation, migration, and invasion of cervical carcinoma cells. We selected the cell lines C33A to investigate a gain-of-function of KISS1 in cervical carcinoma cell lines. KISS1 expression was upregulated after overexpression in C33A cells. The transfection of KISS1 plasmid significantly upregulated the expression of KISS1 in both mRNA and protein levels in C33A (Fig. 4A and B). CCK8 and colony formation assay revealed that overexpression of KISS1 promoted cell proliferation and viability in C33A cells $(P<0.01$, Fig. $4 C-E)$. Besides, cell OD values were significantly reduced at 36 , 48 , and $72 \mathrm{~h}$ in $\mathrm{KISS} 1$ overexpression group (Fig. 4E). The number of cells invasion through the chamber in KISS1 overexpression group was significantly lower than that of the vector group $(P<0.05)$ (Fig. 4F and $\mathrm{G})$. The scratch healing rate of KISS1 overexpressing transfected was significantly lower than that of the vector group $(\mathrm{P}<0.05)(\mathrm{Fig} .4 \mathrm{H})$. The results showed that overexpression of KISS1 inhibited cell migration, invasion and proliferation.

Knockdown of KISS1 on migration and invasion and proliferation of cervical epithelial cells. We selected the cervical epithelial cell line CRL-2614 to investigate the loss-of-function of KISS1 in cervical epithelial cells. The transfection of KISS1 sh significantly downregulated the transcription of KISS1 $(P<0.01$, 
Fig. 5A and B). CCK8 and colony formation assay revealed that loss of KISS1 reduced cell proliferation and viability in CRL-2614 cells $(P<0.01$, Fig. $5 C-E)$. Matrigel invasion and wound healing assays were performed after transfection of KISS1 sh which reduced the transcription and production of KISS1 $(\mathrm{P}<$ 0.01 , Fig. $5 \mathrm{~F}-\mathrm{H}$ ). It can be seen that knockdown of KISS1 enhances cell migration and invasion in cervical epithelial cells.

\section{Discussion}

The signaling pathway in the initial control of tumor cells is activated and the primary tumor cells migrate into nonneoplastic tissues. After the tumor cells are in contact with the blood and lymphatic vessels, penetrate the basement membrane and the endothelial wall, and disperse through the lumen of the blood vessel to reach distant organs, metastasis occurs $[13,14]$. Therefore, exploring tumor metastasis suppressor genes to interfere with specific points in these steps to block the metastatic cascade and prevent metastatic tumor growth is critical for early diagnosis, treatment, and better clinical outcomes. The KISS1 gene was first reported as a novel metastasis suppressor gene in human melanoma and breast cancer cells $[15,16]$. The translation product of KISS1 is a protein of 145 amino acids, which is further cleaved into Kisspeptin-10, Kisspeptin-13, Kisspeptin-14, Kisspeptin-54[17, 18]. KISS1 proteins bind specifically to GPR54 (AXOR12 or hOT7T175) and induces the second messenger inositol trisphosphate (IP3) and diglycerides, which play a role in cell proliferation, differentiation and apoptosis[6, 7].

However, the expression of KISS1 and its pathogenesis in cervical carcinoma remains unclear. To investigate the mechanism of action of KISS1 in cervical carcinoma, promoter methylation and related clinicopathological data in the cervical carcinoma were analyzed. In this research, hypermethylation of KISS1 is significantly present in cervical carcinoma tissues compared to nonneoplastic tissues, indicating that methylation of KISS1 may contribute to the progression in cervical carcinoma. Statistical analysis between the KISS1 promoter methylation and pathological parameters of patients showed that in cervical carcinoma patients with lymph node metastasis and TNM III + IV stage, the methylation positive rate of KISS1 promoter is higher, indicating the complexity of tumor pathogenesis is not only a reflection of genetic change by mutation or deletion but also a reflection of epigenetic alterations, such as DNA methylation. In addition to the deletion and mutation of related genes, aberrant changes in DNA methylation are considered as the third mechanism leading to anti-oncogene inactivation[19,20] which plays an essential role in tumor development. A previous study suggested that hypermethylation of KISS1 was occurred frequently in colorectal cancer[21]. Previous studies have also confirmed that KISS1 methylation is associated with tumor differentiation, depth of invasion, distant metastasis of lymph nodes, and predictive recurrence[9, 21, 22]. These data indicated KISS1 methylation may be associated with invasion, metastasis and poor prognosis in cervical carcinoma. These results are similar to the results of our groups. 
The KISS1 expression is substantially down-regulated in cervical carcinoma tissues compared to nonneoplastic non-tumor tissues according to immunohistochemical staining. This is consistent with the findings of Kostakis that found that KISS1 expression in nonneoplastic cervical mucosa was much higher than in malignant mucosa[23]. Then we statistically analyzed the pathological features of cervical carcinoma tissues, and found that low expression of KISS1 was significantly associated with lymph node metastasis and TNM phase. This is consistent with other studies that found that KISS1 has low expression in colorectal cancer and KISS1 is a putative metastasis suppressor in human colorectal cancer[24-26]. We hypothesized that KISS1 promoter methylation results in loss of expression and metastasis of cervical carcinoma. Previous studies have demonstrated that KISS1 hypermethylation is associated with loss of transcription and protein expression in colorectal cancer (CRC)[9]. Furthermore, promoter $\mathrm{CpG}$ island methylation reduced the expression of related tumor suppressor genes was the main tumor suppressor-inactivation mechanism in cervical carcinoma[27]. Therefore, it would be essential to examine whether KISS1 expression in cervical carcinoma tissues is also directly affected by its promoter methylation. This indicates that the KISS1 protein plays a role in inhibiting tumor metastasis during the development of cervical carcinoma, further confirming that the KISS1 gene is a metastasis suppressor gene in cervical carcinoma, and its down-regulation of expression has certain guiding significance for clinical development of individualized treatment plan and prognosis.

To verify the effect of KISS1 on cervical carcinoma transfer, we examined the biological function of KISS1 on cervical carcinoma cell line C33A compared with those in the normal human cervical epithelial cell line CRL2614. Our data indicate that overexpression of the KISS1 gene significantly inhibits the migration and invasion of C33A in the cervical carcinoma cell line; its decreased expression can promote migration and invasion in CRL2614 cells. These results are similar to those reported by Li et al that KISS1 may inhibit the migration and invasion in BGC-823 gastric carcinoma cells [28]. Chen pointed out KISS1 overexpression significantly decreased the invasiveness of olorectal cancer (CRC) cells [29]. Previous reports have also pointed out that the reduction of KISS1 expression promotes cell migration and invasion in pancreatic [30, 31], ovarian [31], prostate [32], endometrial cancer[33]and nasopharyngeal carcinoma [34]. Considering the importance of migration and invasion ability to tumor progression and metastasis, the results demonstrate the therapeutic potential of KISS1 by reducing the ability of tumor cell metastasis.

Data from proliferation experiments indicate that the overexpressed KISS1 has an inhibitory effect in C33A cells and the knockdown KISS1 has a promoting effect in knockdown CRL2614 cells. These effects appear 48 hours after treatment may because KISS1 takes more time to exert its effect on proliferation. Interestingly, the role of KISS1 in proliferation is various in different tumors. Chen[29]found that the silencing of KISS1 gene had no influence on proliferation, and overexpression of KISS1 led to a significant decrease in the proliferation in HCT-116 colorectal cancer cells. However, knockdown of KISS1 in HT115 and HRT18 colorectal cancer cells has no effect on proliferation [25]. Inconsistent proliferation results may be related to the characteristics of different tumor cells. It has been reported that KISS1 inhibits growth of matrix-independent tumor but not of matrix-dependent tumor[34]. Therefore, the 
regulation of KISS1 in different tumor phenotypes is more complex than expected and requires further investigation.

\section{Conclusion}

Above all, the experiment demonstrated that KISS1, as a tumor metastasis suppressor gene, is closely associated with the development of cervical carcinoma and can inhibit the migration and invasion of cervical carcinoma to a certain extent. Consequently, KISS1 can be used as a new target for clinical treatment, which may not only eliminate local diseases, but also inhibit the systemic spread of cervical carcinoma cells, but requires further research to confirm.

\section{Declarations}

\section{Acknowledgements}

We would like to thank American Journal Experts (AJE) for English language editing.

\section{Funding}

No funding applied.

\section{Availability of data and materials}

The datasets used and/or analyzed during the current study are available from the corresponding author on reasonable request.

\section{Authors' contributions}

YZ designed the study. ZW and MW performed the experiments. HY analyzed the data. HW and CW wrote the manuscript together. JQ and FM helped to revise the manuscript. All authors read and approved the final manuscript and agree to be accountable for all aspects of the research in ensuring that the accuracy or integrity of any part of the work are appropriately investigated and resolved.

\section{Ethics approval and consent to participate}

The study was approved by the Institutional Ethical Review Committee of Jilin Cancer Hospital and adhered to the principles of the Declaration of Helsinki. Informed consent was obtained from each patient before collection of tissues.

\section{Patient consent for publication}

Not applicable.

\section{Competing interests}


The authors declare that they have no competing interests.

\section{References}

1. Monaghan JM: Central recurrent cervical cancer: The role of exenterative surgery. In An Atlas of Gynecologic Oncology. CRC Press; 2018: 102-108

2. Lei J, Andrae B, Ploner A, Lagheden C, Eklund C, Kleppe SN, Wang J, Fang F, Dillner J, Elfström KM: Cervical screening and risk of adenosquamous and rare histological types of invasive cervical carcinoma: population based nested case-control study. bmj 2019, 365:|1207.

3. Chen W, Sun K, Zheng R, Zeng H, Zhang S, Xia C, Yang Z, Li H, Zou X, He J: Cancer incidence and mortality in China, 2014. Chin J Cancer Res 2018, 30:1-12.

4. Samant RS, Seraj MJ, Saunders MM, Sakamaki TS, Shevde LA, Harms JF, Leonard TO, Goldberg SF, Budgeon L, Meehan WJ, et al: Analysis of mechanisms underlying BRMS1 suppression of metastasis. Clin Exp Metastasis 2000, 18:683-693.

5. Lee JH, Miele ME, Hicks DJ, Phillips KK, Trent JM, Weissman BE, Welch DR: KiSS-1, a novel human malignant melanoma metastasis-suppressor gene. J Natl Cancer Inst 1996, 88:1731-1737.

6. Clements MK, McDonald TP, Wang R, Xie G, O'Dowd BF, George SR, Austin CP, Liu Q: FMRFamiderelated neuropeptides are agonists of the orphan G-protein-coupled receptor GPR54. Biochem Biophys Res Commun 2001, 284:1189-1193.

7. Stafford LJ, Xia C, Ma W, Cai Y, Liu M: Identification and characterization of mouse metastasissuppressor KiSS1 and its G-protein-coupled receptor. Cancer Res 2002, 62:5399-5404.

8. Zhang Y, Huang Z, Zhu Z, Zheng X, Liu J, Han Z, Ma X, Zhang Y: Upregulated UHRF1 promotes bladder cancer cell invasion by epigenetic silencing of KiSS1. PLOS One 2014, 9:e104252.

9. Moya P, Esteban S, Fernandez-Suarez A, Maestro M, Morente M, Sanchez-Carbayo M: KiSS-1 methylation and protein expression patterns contribute to diagnostic and prognostic assessments in tissue specimens for colorectal cancer. Tumour Biol 2013, 34:471-479.

10. Teng Y, Mei Y, Hawthorn L, Cowell JK: WASF3 regulates miR-200 inactivation by ZEB1 through suppression of KISS1 leading to increased invasiveness in breast cancer cells. Oncogene 2014, 33:203-211.

11. Zhang X, Wang X, Wang A, Li Q, Zhou M, Li T: CLDN10 promotes a malignant phenotype of osteosarcoma cells via JAK1/Stat1 signaling. J Cell Commun Signal 2019, 13:395-405.

12. Zhang $X$, Wang H, Li Q, Li T: CLDN2 inhibits the metastasis of osteosarcoma cells via downregulating the afadin/ERK signaling pathway. Cancer Cell Int 2018, 18:160.

13. Hou JM, Krebs M, Ward T, Sloane R, Priest L, Hughes A, Clack G, Ranson M, Blackhall F, Dive C: Circulating tumor cells as a window on metastasis biology in lung cancer. Am J Pathol 2011, 178:989-996.

14. Kienast $Y$, von Baumgarten L, Fuhrmann M, Klinkert WE, Goldbrunner R, Herms J, Winkler F: Real-time imaging reveals the single steps of brain metastasis formation. Nat Med 2010, 16:116-122. 
15. Lee JH, Welch DR: Suppression of metastasis in human breast carcinoma MDA-MB-435 cells after transfection with the metastasis suppressor gene, KiSS-1. Cancer Res 1997, 57:2384-2387.

16. Lee JH, Welch DR: Identification of highly expressed genes in metastasis-suppressed chromosome 6/human malignant melanoma hybrid cells using subtractive hybridization and differential display. Int J Cancer 1997, 71:1035-1044.

17. Kotani M, Detheux M, Vandenbogaerde A, Communi D, Vanderwinden JM, Le Poul E, Brezillon S, Tyldesley R, Suarez-Huerta N, Vandeput F, et al: The metastasis suppressor gene KiSS-1 encodes kisspeptins, the natural ligands of the orphan G protein-coupled receptor GPR54. J Biol Chem 2001, 276:34631-34636.

18. Ohtaki T, Shintani Y, Honda S, Matsumoto H, Hori A, Kanehashi K, Terao Y, Kumano S, Takatsu Y, Masuda Y, et al: Metastasis suppressor gene KiSS-1 encodes peptide ligand of a G-protein-coupled receptor. Nature 2001, 411:613-617.

19. Fang JY, Lu YY: Effects of histone acetylation and DNA methylation on p21(WAF1) regulation. World J Gastroenterol 2002, 8:400-405.

20. Kumar R, Xi Y: MicroRNA, epigenetic machinery and lung cancer. Thorac Cancer 2011, 2:35-44.

21. Chen SQ, Chen ZH, Lin SY, Dai QB, Fu LX, Chen RQ: KISS1 methylation and expression as predictors of disease progression in colorectal cancer patients. World J Gastroenterol 2014, 20:10071-10081.

22. Cebrian V, Fierro M, Orenes-Piñero E, Grau L, Moya P, Ecke T, Alvarez M, Gil M, Algaba F, Bellmunt J, et al: KISS1 methylation and expression as tumor stratification biomarkers and clinical outcome prognosticators for bladder cancer patients. Am J Pathol 2011, 179:540-546.

23. Kostakis ID, Agrogiannis G, Vaiopoulos AG, Mylona E, Patsouris E, Kouraklis G, Koutsilieris M: KISS1 and KISS1R expression in gastric cancer. J buon 2018, 23:79-84.

24. Kostakis ID, Agrogiannis G, Vaiopoulos AG, Mylona E, Patsouris E, Kouraklis G, Koutsilieris M: A clinicopathological analysis of KISS1 and KISS1R expression in colorectal cancer. Apmis 2015, 123:629-637.

25. Ji K, Ye L, Ruge F, Hargest R, Mason MD, Jiang WG: Implication of metastasis suppressor gene, Kiss1 and its receptor Kiss-1R in colorectal cancer. BMC Cancer 2014, 14:723.

26. Huo X, Zhang L, Li T: Analysis of the association of the expression of KiSS-1 in colorectal cancer tissues with the pathology and prognosis. Oncol Lett 2018, 15:3056-3060.

27. Guan Z, Zhang J, Song S, Dai D: Promoter methylation and expression of TIMP3 gene in gastric cancer. Diagn Pathol 2013, 8:110.

28. Li N, Wang HX, Zhang J, Ye YP, He GY: KISS-1 inhibits the proliferation and invasion of gastric carcinoma cells. World J Gastroenterol 2012, 18:1827-1833.

29. Chen S, Chen W, Zhang X, Lin S, Chen Z: Overexpression of KiSS-1 reduces colorectal cancer cell invasion by downregulating MMP-9 via blocking PI3K/Akt/NF-kappaB signal pathway. Int J Oncol 2016, 48:1391-1398. 
30. Wang CH, Qiao C, Wang RC, Zhou WP: KiSS1mediated suppression of the invasive ability of human pancreatic carcinoma cells is not dependent on the level of KiSS1 receptor GPR54. Mol Med Rep 2016, 13:123-129.

31. Jiang Y, Berk M, Singh LS, Tan H, Yin L, Powell CT, Xu Y: KiSS1 suppresses metastasis in human ovarian cancer via inhibition of protein kinase C alpha. Clin Exp Metastasis 2005, 22:369-376.

32. Wang H, Jones J, Turner T, He QP, Hardy S, Grizzle WE, Welch DR, Yates C: Clinical and biological significance of KISS1 expression in prostate cancer. Am J Pathol 2012, 180:1170-1178.

33. Kang HS, Baba T, Mandai M, Matsumura N, Hamanishi J, Kharma B, Kondoh E, Yoshioka Y, Oishi S, Fujii N, et al: GPR54 is a target for suppression of metastasis in endometrial cancer. Mol Cancer Ther 2011, 10:580-590.

34. Wang GM, Liu JF, Zhang L, Sun Q, Zhou Y, Xu HB, Zhang YJ, Cai F, Cheng ZN, Xiang P, Jiang H: Inhibition of KiSS-1 on metastasis of nasopharyngeal carcinoma implant tumor in nude mice. $\mathrm{Am} \mathrm{J}$ Transl Res 2019, 11:904-910.

\section{Tables}

Table 1. Association between clinicopathological features and methylation in 97 patients with cervical carcinoma 


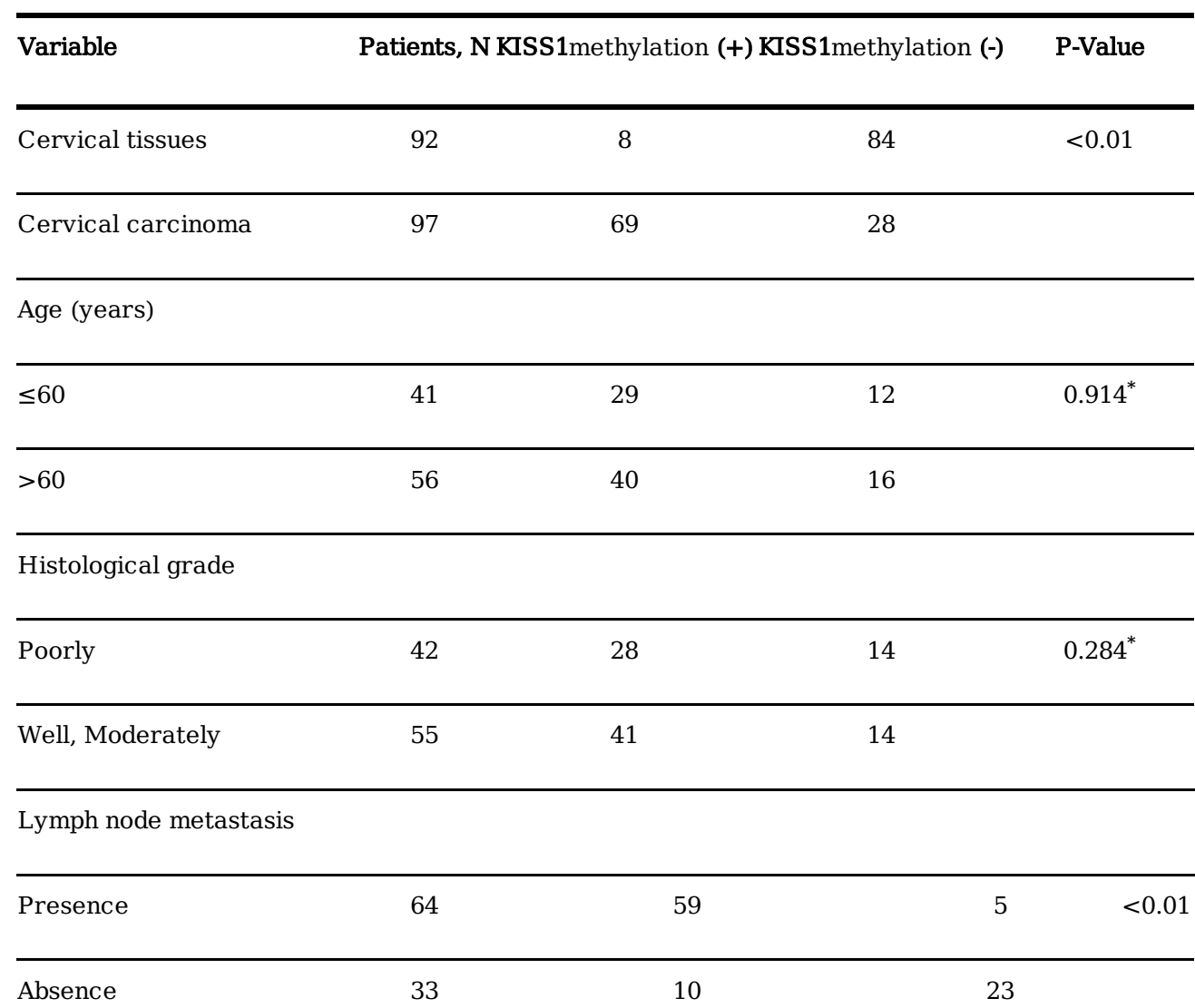

\begin{tabular}{lcccc} 
TNM stage (AJCC) & & & & \\
\hline I-II & 39 & 27 & 22 & $<0.01$ \\
\hline III-IV & 58 & 52 & 6 & \\
\hline
\end{tabular}

${ }^{*}$ No statistical significance was found with the $\chi 2$ test/ $\chi 2$ Goodness-of-Fit Test. Kisspeptin, KISS1. TNM, Tumor-Node-Metastasis.

Table 2. Relationship between expression of KISS-1 and clinicopathological variables in 97 patients with cervical carcinoma 


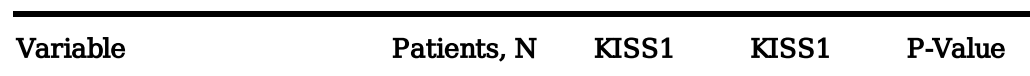

$(+) \quad(-)$

\begin{tabular}{lcccc}
\hline Cervical tissues & 92 & 81 & 11 & $<0.01$ \\
\hline Cervical carcinoma & 97 & 24 & 73 & \\
\hline Age (years) & & & & \\
\hline$\leq 60$ & 41 & 13 & 28 & $0.134^{*}$ \\
\hline$>60$ & 56 & 11 & 45 & \\
\hline Histological grade & & & & \\
\hline Poorly & & & & \\
& 42 & 12 & 30 & $0.296^{*}$ \\
\hline Well, Moderately & 55 & 12 & 14 & \\
\hline
\end{tabular}

Lymph node metastasis

\begin{tabular}{lcccc}
\hline Presence & 64 & 8 & 56 & $<0.01$ \\
\hline Absence & 33 & 16 & 17 &
\end{tabular}

TNM stage (AJCC)

\begin{tabular}{lcccc}
\hline I-II & 39 & 18 & 21 & $<0.01$ \\
\hline III-IV & 58 & 6 & 52 & \\
\hline
\end{tabular}

${ }^{*}$ No statistical significance was found with the $\chi 2$ test/ $\chi 2$ Goodness-of-Fit Test. Kisspeptin, KISS1. TNM, Tumor-Node-Metastasis.

\section{Figures}




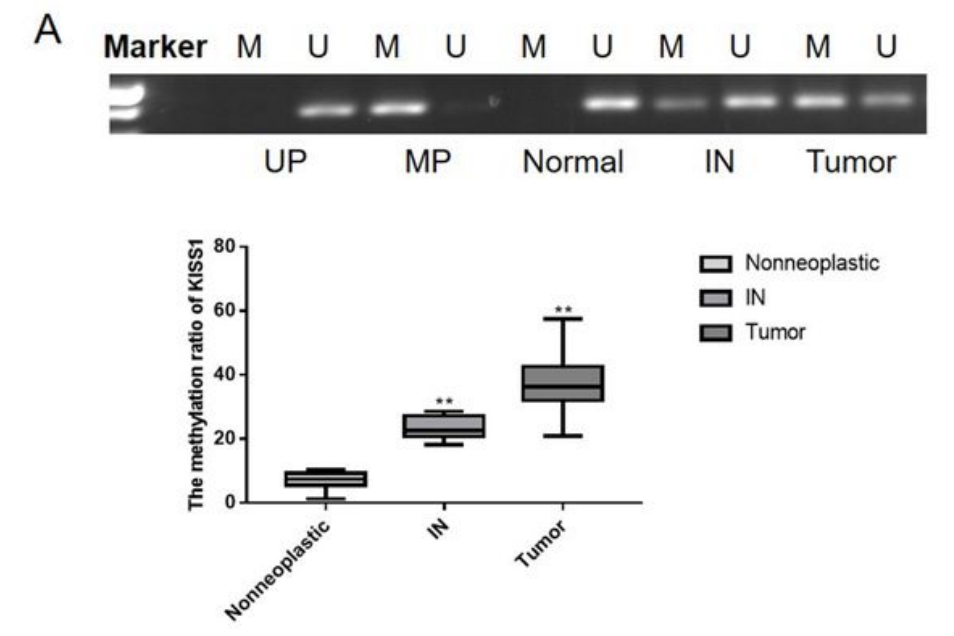

B
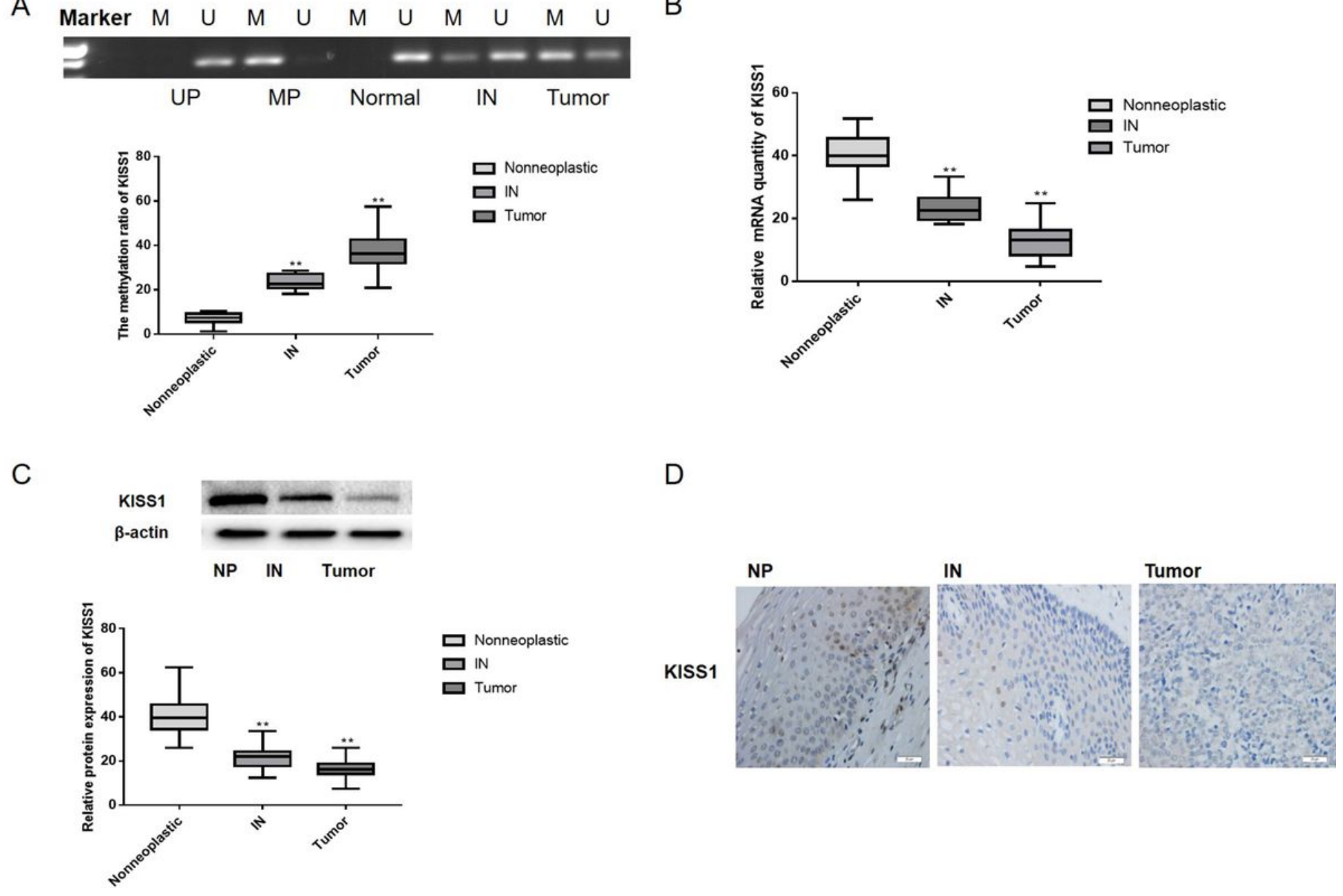

\section{Figure 1}

Expression of KISS1 in cervical tumor and nonneoplastic tissues. (A)The methylation status of KISS1 in cervical carcinoma tissues, intraepithelial neoplasia (IN) tissues and normal cervical tissues. M: methylation; U: unmethylation; Up: Unmethylated positive contrast; MP: Methylated positive contrast; intraepithelial neoplasia, IN. (B) The mRNA expression of KISS1 was performed with real-time qPCR in cervical carcinoma tissues, IN tissues and normal cervical tissues. (C) The protein expression of KISS1 was performed with western-blotting in cervical carcinoma tissues, IN tissues and normal cervical tissues. (D) The expression of KISS1 was performed with IHC staining in cervical carcinoma tissues, IN tissues and normal cervical tissues. Original magnification: $\times 200 .{ }^{*} P<0.05,{ }^{*} P<0.01$. Kisspeptin, KISS1. 


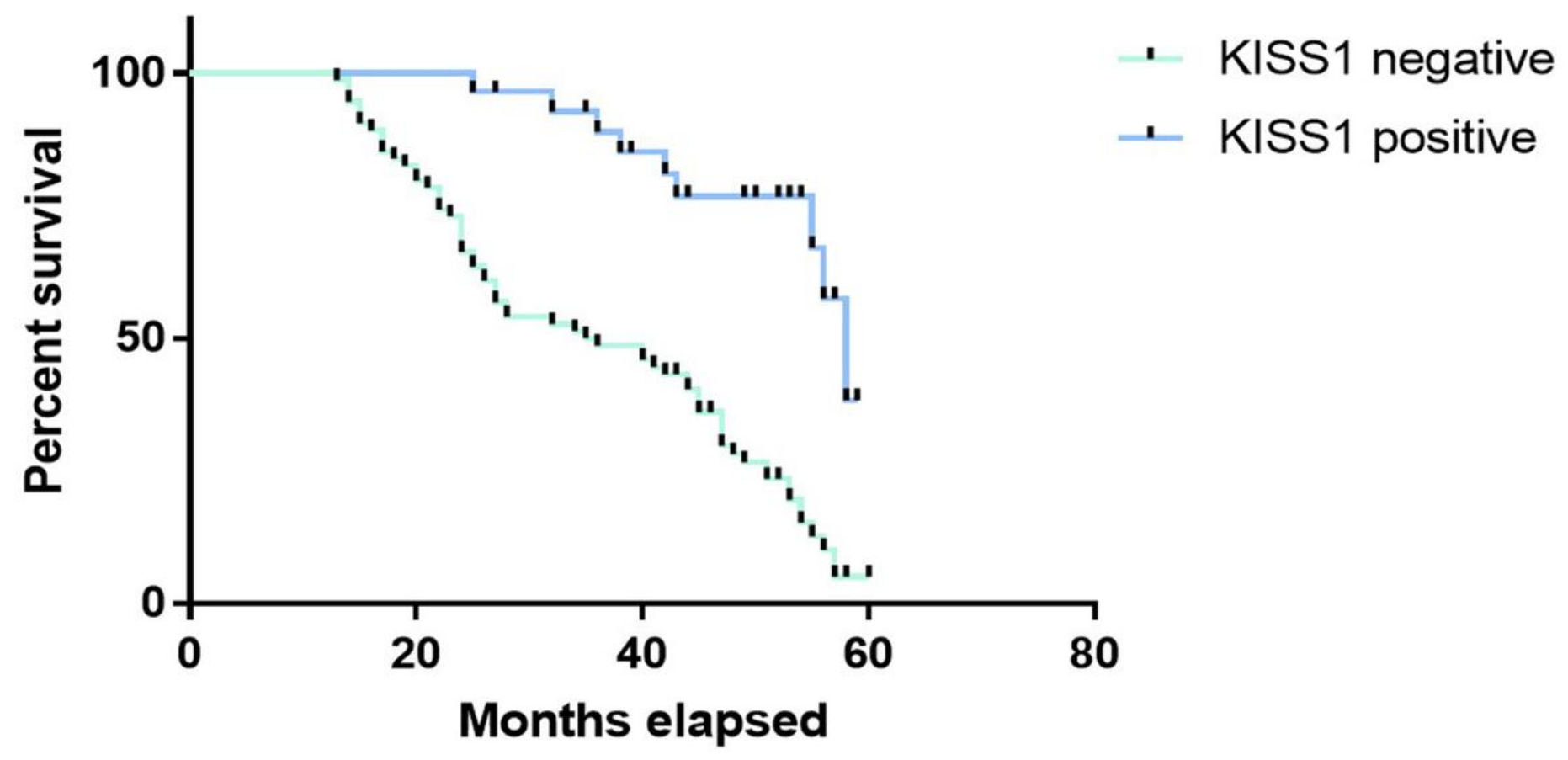

Figure 2

Associations between KISS1 expression and the prognosis of patients with cervical carcinoma. ${ }^{\star \star} \mathrm{P}<0.01$. Kisspeptin, KISS1.

A

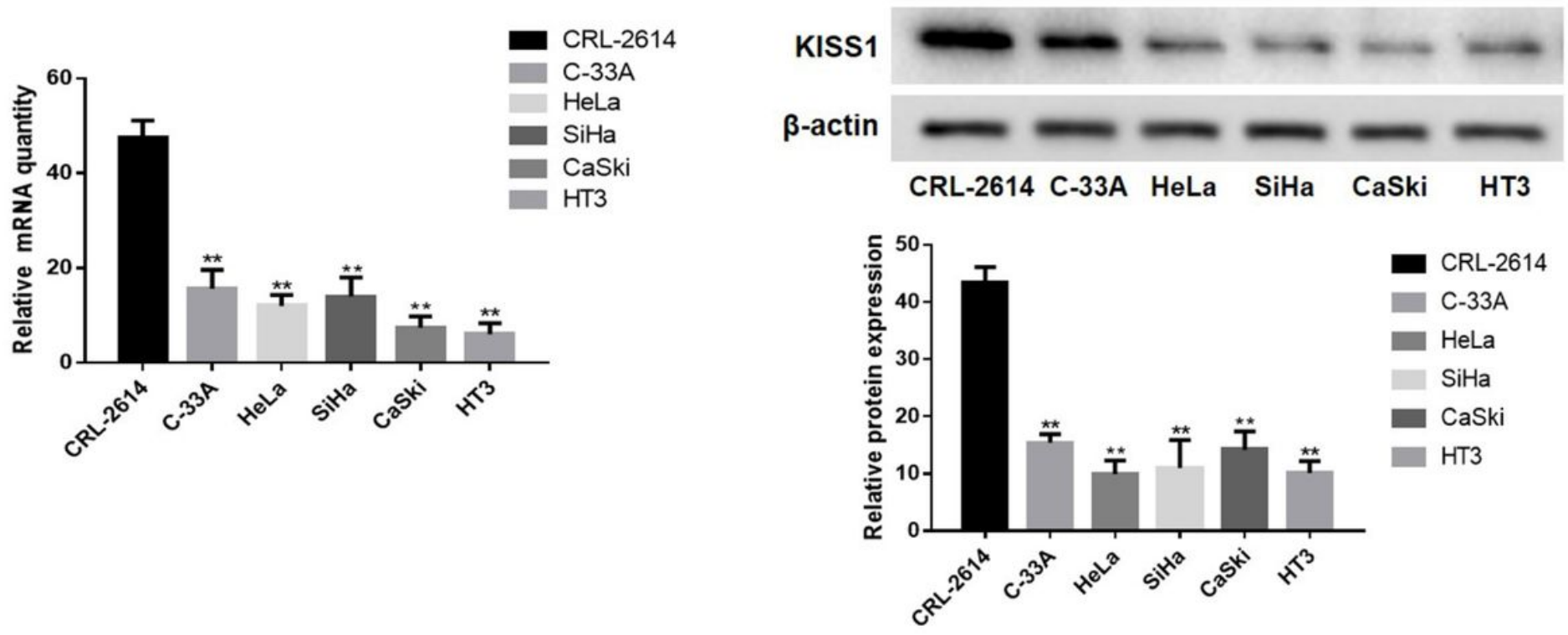

Figure 3

KISS1 was downregulated in cervical carcinoma cell lines. (A) The expression levels of KISS1 were assessed in cervical carcinoma cell lines (C-33A, HeLa, SiHa, CaSki, HT3) and cervical epithelial cell line 
(CRL-2614) by RT-qPCR. (B) The western blotting results revealed that KISS1 was expressed at a lower level in the cervical carcinoma cell lines compared with the CRL2614 cell line. ${ }^{*} P<0.01$. Kisspeptin, KISS1.

A

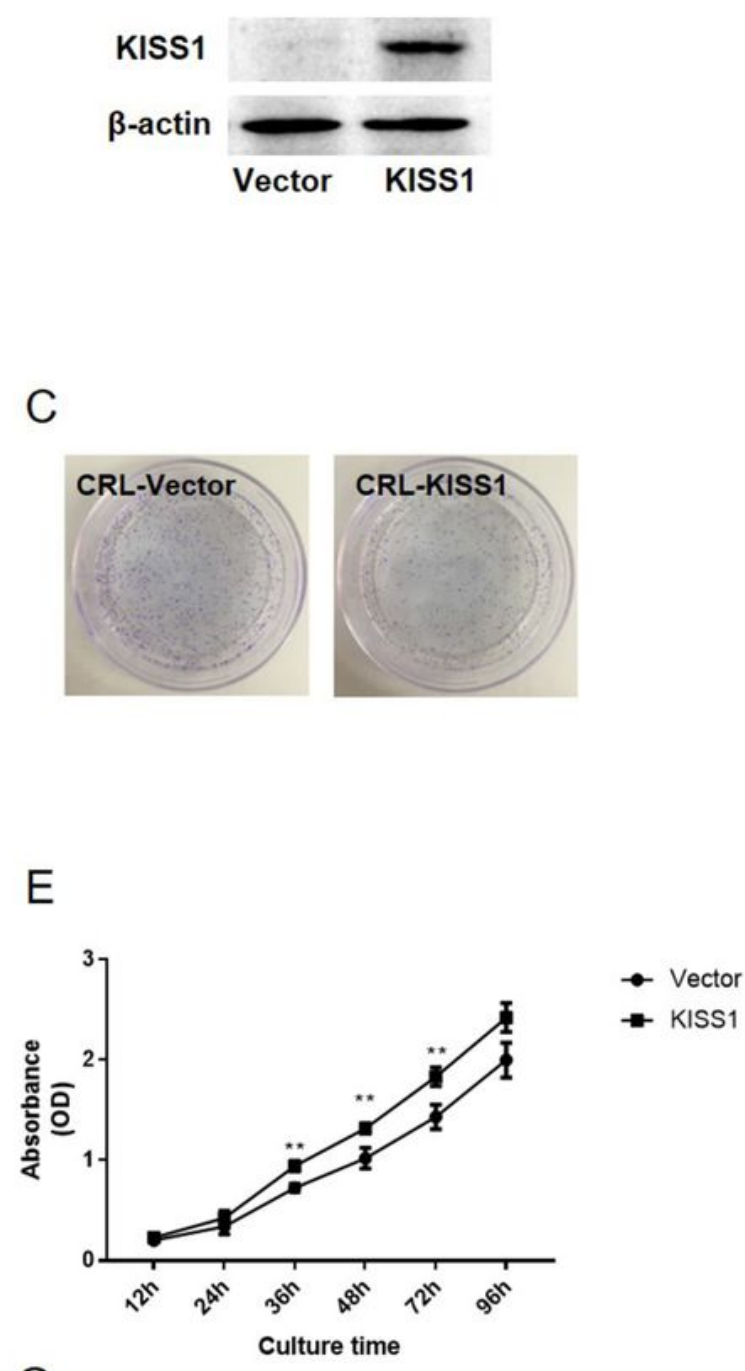

G

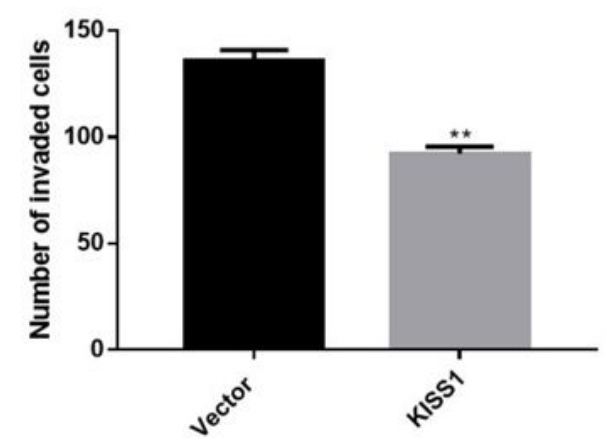

B

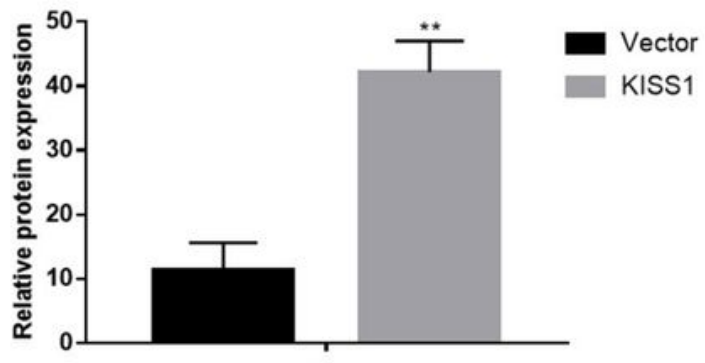

D

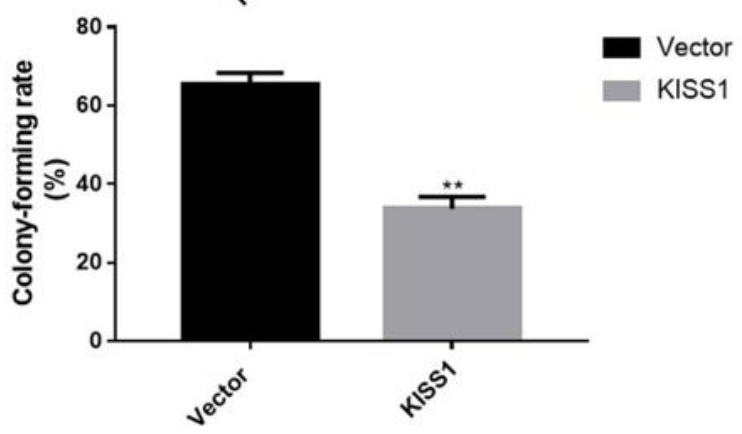

$\mathrm{F}$

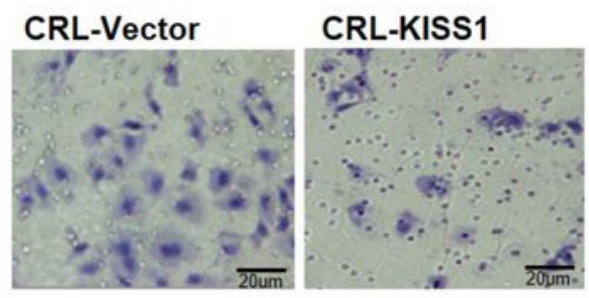

$\mathrm{H}$

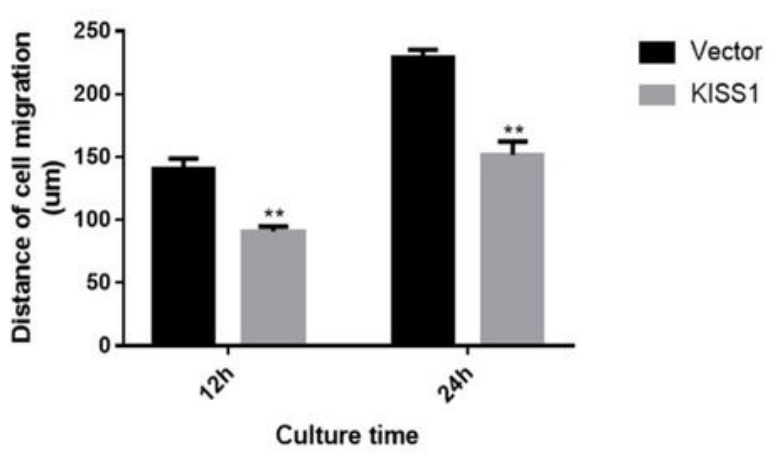

Figure 4

The effect of KISS1 overexpression on cervical carcinoma cells was examined. (A) Illustration of western blot after KISS1 plasmid transfection. (B) The KISS expression level of KISS1 plasmid transfection group 
was significantly different from control group. ${ }^{*} \mathrm{P}<0.01$. (C) Representative image of KISS1 overexpressing cells on cell colony formation ability; (D) The cell colony formation ability of KISS1 overexpressing cells was significantly different from control group. ${ }^{*} \mathrm{P}<0.01$. (E) Effects of KISS1 overexpression on cell growth using CCK8 assay. ${ }^{\star *} \mathrm{P}<0.01$. (F) Representative image of KISS1 overexpressing cells invades the chamber membrane; (G) The mean number of invaded cells in control, overexpressing and KISS1 overexpressing groups; $(\mathrm{H})$ Quantitative analysis of wound closure in control, overexpressing and KISS1 overexpressing groups. ** $\mathrm{P}<0.01$. ${ }^{* *} \mathrm{P}<0.01$, Kisspeptin, KISS1.

A

KISS1

$\beta$-actin

Scramble shRNA1 shRNA2

C

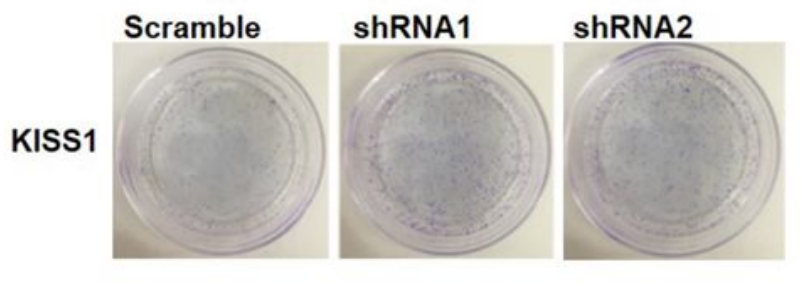

E

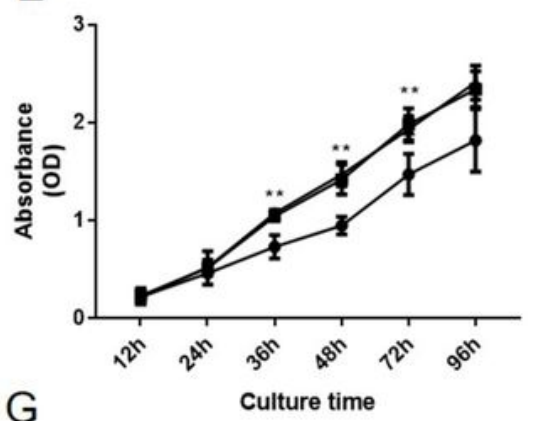

G

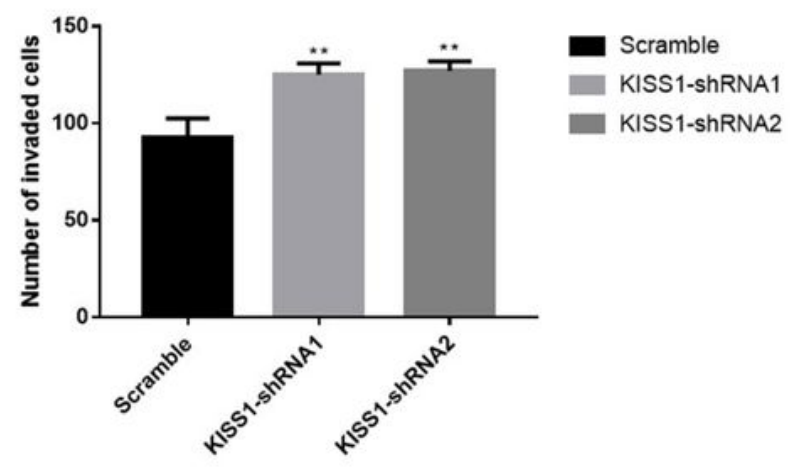

B
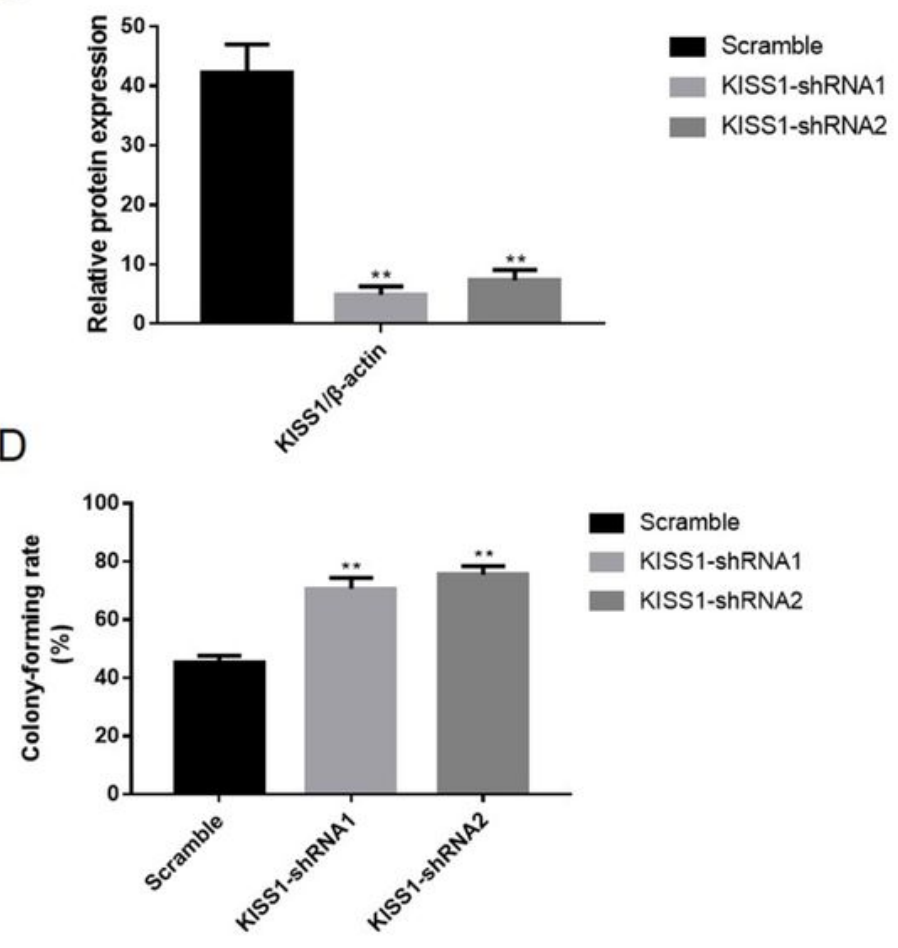

F

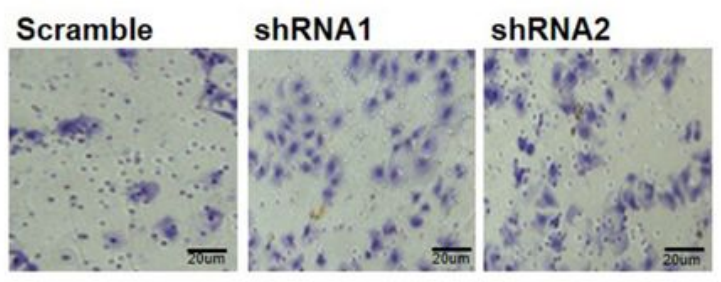

$\mathrm{H}$

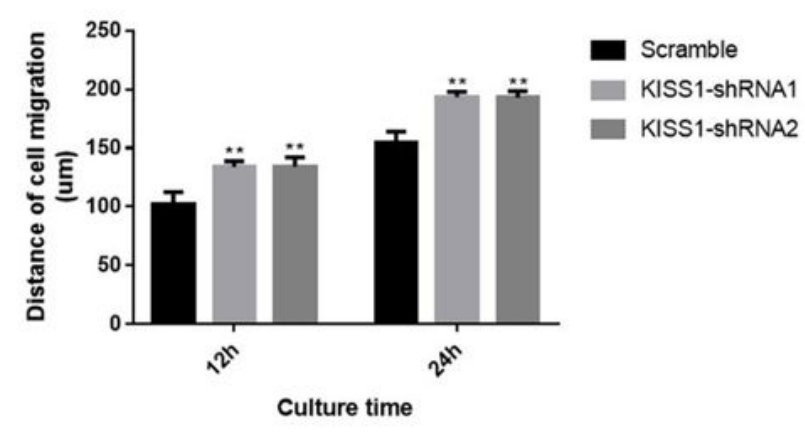




\section{Figure 5}

The effect of KISS1 knockdown on cervical carcinoma cells was examined. (A) Illustration of western blot after KISS1 sh transfection. (B) The KISS expression level of KISS1 sh transfection group was significantly different from scramble group (**P<0.01). (C) Representative image of KISS1 knockdown cells on cell colony formation ability; (D) The cell colony formation ability of KISS1 knockdown cells was significantly different from scramble group $\left({ }^{*} P<0.01\right)$. (E) Effects of KISS1 knockdown on cell growth using CCK8 assay. ${ }^{*} P<0.05$. (F) Representative image of KISS1 knockdown cells invades the chamber membrane; $(G)$ The mean number of invaded cells in scramble and KISS1 sh groups. ${ }^{\star *} P<0.01 ;(H)$ Quantitative analysis of wound closure in scramble and KISS1 sh groups. ${ }^{* *} \mathrm{P}<0.01$, Kisspeptin, KISS1. 